\title{
Identification of the Translation Start Site of the Human Melanocortin 3 Receptor
}

\author{
Patrick Tarnow $^{a} \quad$ Anne Rediger ${ }^{a} \quad$ Angela Schulz $^{b} \quad$ Annette Grüters ${ }^{a}$ \\ Heike Biebermann ${ }^{a}$ \\ anstitute of Experimental Pediatric Endocrinology, Charité - Universitätsmedizin Berlin, Humboldt \\ University, Berlin, ${ }^{b}$ Institute of Biochemistry, Medical Faculty, University of Leipzig, Leipzig, Germany
}

\author{
Key Words \\ Melanocortin · Obesity · Polymorphism · Translation
}

\begin{abstract}
Background: The melanocortin-3-receptor (MC3R) is a G-protein coupled receptor participating in hypothalamic energy metabolism. So far, it was assumed that the translation of the human MC3R starts at the non-conserved first ATG, however, a second evolutionary conserved ATG is located 37 amino acids downstream. One frequent polymorphism, T6K, is located between these two ATGs. Methods: For characterization of the two potential start ATGs, COS-7 cells were transfected with plasmids encoding the longer and the shorter form of the human MC3R. For signal transduction properties, CAMP was measured. Cell surface expression was determined by using an ELISA method. The translational start point of the MC3R was investigated by a GFP-based method. Results: Signal transduction was comparable for the long and the short receptor form. Cell surface expression via aminoterminal hemagglutinin tag could only be detected in the shorter form, but not in the longer one. In our study we show that the translation of the human MC3R protein starts at the evolutionary conserved ATG codon which results in a shorter protein than previously assumed. Conclusion: The polymorphism T6K is not located in the coding region of the human MC3R and has no influence on translation initiation which makes an impact on body weight unlikely.
\end{abstract}

Copyright (c) 2012 S. Karger GmbH, Freiburg

\section{Introduction}

Melanocortin receptors are rhodopsin-like G-protein-coupled receptors, which are activated by the processing products of pro-opiomelanocortin. Both the melanocortin-3receptor (MC3R) and the melanocortin-4-receptor (MC4R) are expressed in the hypothalamus and are in involved in energy metabolism and food intake, respectively. Genetic disruption of the $M C 4 R$ gene leads to a hyperphagic and obese phenotype in mice [1], whereas 
disruption of the $M C 3 R$ gene leads to hypophagia and an increased fat mass but to normal body weight $[2,3]$. Mutations in the $M C 4 R$ are the most frequent cause for monogenetic obesity in humans [4]. In contrast, the role of mutations in the human MC3R contributing to obesity is currently being investigated [5]. So far, only few MC3R mutations with functional relevance were identified [6-9]. However, numerous studies investigated the association of two frequent $M C 3 R$ variants with obesity or diabetes in various collectives [10-14]. These variants are located in the coding region and lead to amino acid exchanges T6K (nt C17A) and V81I (nt G241A) and the double mutant T6K/V81I. In most studies, no associations between MC3R variants and obesity or diabetes could be found. However, in children, homozygous carriers of the T6K/V81I haplotype have been shown to have a higher BMI and more body fat [14], to consume more calories [15], and to lose less weight in a weight loss program [16]. In vitro investigation of signal transduction capabilities suggested that these mutations do not have an impact on receptor function [17]; however, a partial inactivation was shown for the double mutant T6K/V81I [14]. By comparing published orthologous MC3R sequences we show in our study that only human and chimpanzee MC3R exhibit an extended amino terminus introducing an additional ATG codon in the $5^{\prime}$ region (further referred to as ATG1) according to MC3R sequences of other species. So far, ATG1 is thought to be the start codon for humans, and amino acid numbering is according to ATG1. There is a second ATG 37 amino acid downstream of ATG1 (further referred to as ATG2) which is the start ATG for all other species (fig. 1A, B). Nonetheless, in COS-7 cells we can show that ATG2 in humans is the preferred translation initiation site independently from the T6K variant which is located between the two ATGs.

\section{Material and Methods}

MC3R Cloning, Cell Culture and Functional Characterization

The coding region of the human MC3R was PCR amplified and cloned into the eucaryotic expression vector pcDps. To investigate cell surface expression a hemagglutinine (HA) tag was introduced after the first (ATG1) or the second ATG (ATG2).

For functional characterization COS-7 cells were cultured in Dulbecco's modified medium (DMEM) with $10 \%$ fetal calf serum (FCS), and $20 \mathrm{mmol} / \mathrm{l}$ glutamine. Cells were incubated at $37^{\circ} \mathrm{C}$ in humidified air containing $5 \% \mathrm{CO}_{2}$. Transfections were carried out using Metafectene (Biontex, Munich, Germany) according to the manufacturer's protocol.

Cell surface expression assays were performed in 48 -well plates $\left(4 \times 10^{4}\right.$ cells/well), and cells were transfected with $0.25 \mu \mathrm{g}$ DNA/well and $1 \mu \mathrm{l}$ Metafectene/well with N-terminal HA-tagged vectors (amino acid sequence: YPYDVPDYA).

$72 \mathrm{~h}$ after transfection cells were washed two times with Dulbecco's phosphate buffered saline (DPBS, Biochrom, Berlin, Germany) and fixed for $30 \mathrm{~min}$ in $4 \%$ formaldehyde in DPBS followed by two times washing in DPBS. After incubation in blocking buffer (10\% FCS supplemented DMEM) for $1 \mathrm{~h}$ at $37^{\circ} \mathrm{C}$ followed by a washing step in DPBS, cells were incubated for $2 \mathrm{~h}$ in blocking buffer with $1 \mu \mathrm{g} / \mathrm{ml}$ biotinlabeled anti-HA monoclonal antibody (Roche, Mannheim, Germany) at $37^{\circ} \mathrm{C}$ followed by three washes in DPBS and incubation in blocking buffer with $1 \mu \mathrm{g} / \mathrm{ml}$ streptavidin-labeled peroxidase (Dianova, Hamburg, Germany) at $37^{\circ} \mathrm{C}$ for $1 \mathrm{~h}$ followed by three times washing. The color reaction was carried out with $0.1 \%$ $\mathrm{H}_{2} \mathrm{O}_{2}$ and $10 \mu \mathrm{g} 0$-phenylendiamine in $0.1 \mathrm{~mol} / \mathrm{l}$ citric acid and $0.1 \mathrm{~mol} / \mathrm{l} \mathrm{Na} \mathrm{HPO}_{4}$ at $\mathrm{pH}$ 5.2. The reaction was stopped after $10 \mathrm{~min}$ with $1 \mathrm{~mol} / 1 \mathrm{Na}_{2} \mathrm{SO}_{3}$ in $1 \mathrm{~mol} / \mathrm{l} \mathrm{HCl}$. Colorimetry was carried out using an anthos reader 2001 (anthos labtech instruments, Salzburg, Austria). In this protocol, cells were not permeabilized allowing detection of tagged receptors on the cell surface.

cAMP assays were performed in 48 -well plates $\left(5 \times 10^{4}\right.$ cells/well). Cells were transfected with 0.08 $\mu \mathrm{g}$ DNA/well and $0.93 \mu \mathrm{l}$ Metafectene/well. $48 \mathrm{~h}$ after transfection, cells were washed and stimulated with increasing amounts of NDP-alpha-MSH in the presence of $1 \mathrm{mmol} / \mathrm{l}$ 3-isobutyl-1-methylxanthine for 30 min. The reaction was stopped, and intracellular cAMP was determined based on the AlphaScreen technology (Perkin Elmer Life Science, Inc., Boston, MA, USA) according to the manufacturer's protocol. 


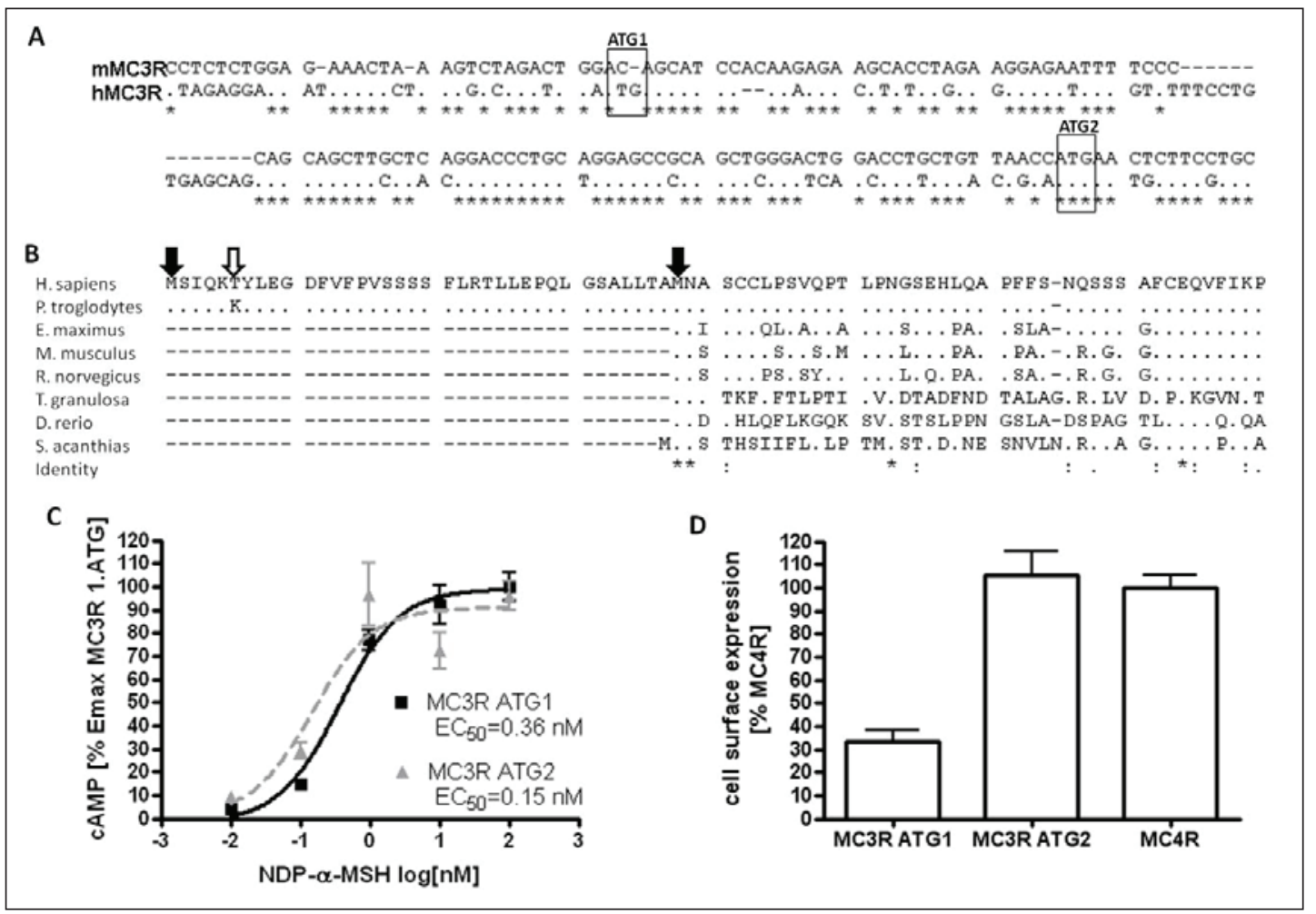

Fig. 1. A DNA alignment of the sequences surrounding the start ATGs of the human and the murine MC3R. For alignments a Clustal W algorithm in the GENtle software was used. Bases identical to the human sequence are indicated as dots, missing amino acids are indicated as bars. The ATG codons are indicated as boxes. B Amino acid alignment of the amino-terminal region of orthologous MC3R. Sequences were derived from the NCBI database: AA072726: Homo sapiens; XP_525362: Pan troglodytes; ABG37016: Elephas maximus; NP_032587: Mus musculus; NP_001020441: Rattus norvegicus; AAS66720: Squalus acanthias; AAI62747: Danio rerio; AAX18229: Taricha granulosa. Amino acids identical to the human sequence are indicated as dots, missing amino acids are indicated as bars. The two start-methionines (filled arrows) and the position of the T6K polymorphism (open arrow) are indicated. C cAMP response of MC3R constructs after stimulation with NDP- $\alpha$-MSH. $48 \mathrm{~h}$ after transfection, COS-7 cells were stimulated with indicated ligand concentrations for $30 \mathrm{~min}$. Cells were lysed and cAMP was measured using the alpha-screen method. Data represent three independent experiments each performed in triplicates. Data were normalized to the maximal response of the MC3R-ATG-1 (49-123 nmol/l cAMP). D Cell surface expression of amino-terminally HA-tagged MC3R and MC4R constructs. $48 \mathrm{~h}$ after transfection cells were fixed with formaldehyde and the extracellular HA epitope was detected immunologically. Optical density was measured photometrically. Background signal of GFP-transfected cells was subtracted and data were normalized to the MC4R signal (OD: 1.00-0.62). Data represent three independent experiments each performed in quadruplicates.

Investigation of the Preferred Start Codon

To investigate the ATG codons in their natural context, we cloned a fragment starting 90 bases upstream the first ATG to 90 bases downstream the second ATG in frame amino-terminal to a GFP cDNA lacking the start ATG into the pcDps vector (fig. 2A). The cloned fragment of the MC3R coding region represents the MC3R amino terminus. Using site-directed mutagenesis based on the quick-change mutagenesis kit (Stratagene, La Jolla, CA, USA), we sequentially mutated the ATG codons to TTG codons yielding constructs with both potential start codons (ATG1/ATG2), one start codon, either ATG1 (ATG1/TTG2) or ATG2 (TGG1/ATG2) or no start codons (TTG1/TTG2). Fluorescence was measured $48 \mathrm{~h}$ after transfection into COS-7 cells. Results were normalized to C-terminally Flag-tagged MC3R; amino acid sequence of C-Flag: DYKDDDDK. 


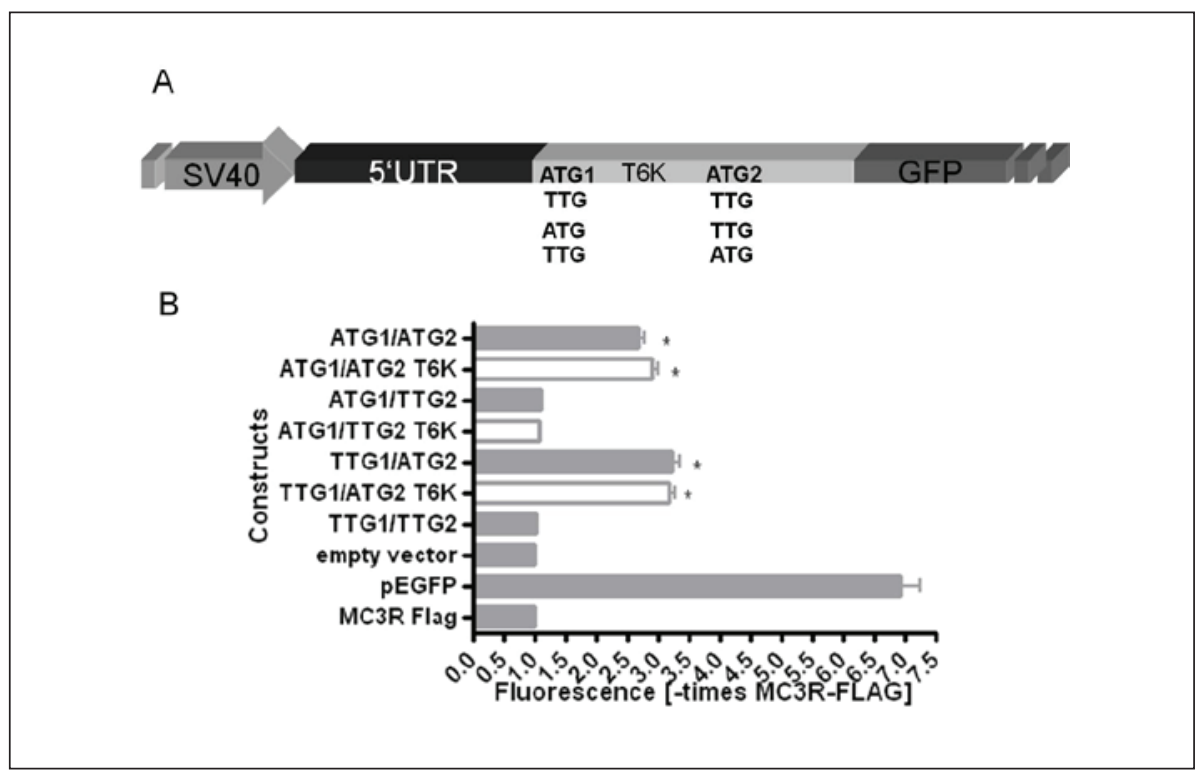

Fig. 2. A Schematic representation of the constructs used in the GFP-reporter gene assay. Following the SV40 promoter encoded by the vector, a fragment including the 5' UTR (black) and the coding sequence of the amino terminus of the hMC3R (light grey) were fused in-frame to the GFP cDNA (grey). Base changes of the ATGs induced by mutagenesis are indicated below. The location of the T6K variant between the ATGs is indicated. B Reporter gene-based translation assay. $48 \mathrm{~h}$ after transfection GFP was measured photometrically. Measured absorption was normalized to an undetectable receptor variant, the MC3R-FLAG construct (amino acid sequence C-Flag: DYKDDDDK). The fluorescence of empty vector and GFP-transfected cells was 60-79 and 301-620, respectively. Filled bars indicate the $\mathrm{T}^{6}$, open bars the $\mathrm{K}^{6}$ variant of the corresponding constructs. ${ }^{*} \mathrm{p}<0.01$ versus empty vector (one-way ANOVA with post-hoc tukey test).

\section{Results}

By investigation of functional properties of the human wild-type MC3R, we initially observed a low cell surface expression of the human MC3R (MC3R ATG1) compared with the human MC4R although both receptors were equally amino-terminally labeled with a HA tag and transiently expressed in COS-7 cells (fig. 1C). Despite this obviously low cell surface expression, signaling of the tagged MC4R and MC3R constructs was comparable. The maximal response $\left(E_{\max }\right)$ of the MC3R ATG1 construct reached $101 \pm 21 \%$ of the MC4R $E_{\max }$ (mean of 3 independent experiments \pm SD, data not shown).

In our previous studies, expression levels of murine MC3R and MC4R were identical [18]. Thus, we aligned orthologous MC3R amino acid sequences published on the NCBI database. According to these sequences the human and the chimpanzee MC3R exhibit an extended amino terminus compared to the other species (fig. 1A, B) as well as to their paralogous MC4R (data not shown). Interestingly, the chimpanzee MC3R has a Lys at amino acid position 6 as wild type (open arrow in fig. 1B).

We amino-terminally HA-tagged the human MC3R at the ATG2 and investigated cell surface expression and cAMP signaling of the shortened construct. As shown in fig. 1C, no differences could be observed in the $\mathrm{E}_{\max }$ or in the half maximal effective concentration $\left(\mathrm{EC}_{50}\right)$ between the two constructs after stimulation with NDP- $\alpha$-MSH. The ATG2-MC3R is expressed at the cell surface to equal levels as the MC4R (fig. 1D). Compared to the ATG1MC3R, a three times higher cell surface expression could be observed for ATG2, suggesting 
that either the HA tag of ATG1 is cleaved off during trafficking to the cell surface or that the HA tag is not translated in the ATG1 construct and translation starts at the ATG2. To verify the latter hypothesis, we chose a green fluorescence protein(GFP)-based reporter assay to investigate translation efficiency [19]. As a positive control, a pEGFP vector was used. Compared with the empty vector significant higher fluorescence was measured for the (ATG1/ATG2) and the (TTG1/ATG2) constructs (fig. 2B). However, in the (TTG1/TTG2) constructs and in the (ATG1/TTG2) constructs no GFP fluorescence could be observed, suggesting that no translation occurred. To investigate if the T6K polymorphism influences the preference of the translation initiation site, this variant was introduced by site-directed mutagenesis. In none of the constructs the K6 variant differed from the corresponding T6 variant in the reporter based translation assay (fig. 2B, open bars) indicating that the T6K variant has no effect on translational efficiency. Because of the non-coding character of this SNP, it should be named by his nucleotide change C17A.

\section{Discussion}

So far it was believed that ATG1 gives rise to a 360 amino acid-long version of the human MC3R if this ATG was used for translation. In this report we provide evidence that not ATG1 but ATG2 is the preferred start ATG for the human MC3R, resulting in a 37 amino acid shorter protein at least in COS-7 cells. This finding is of importance since the association of single nucleotide polymorphisms (SNP) in the coding region of the MC3R with obesity is discussed [10-14]. One of these SNPs, T6K, is located between these two ATGs. Our findings show that T6K is located in the $5^{\prime}$ untranslated region of the human MC3R and has no influence on MC3R translation.

In functional studies the longer variant (ATG1) is frequently used [5, 14, 17]. In our cAMP accumulation experiments the choice of the ATG had no influence on signal transduction properties. In contrast to these results only low amounts of HA-tagged MC3R at the first ATG could be detected on the cell surface, suggesting that the tag could be over-read by the translation machinery. In in vitro assays we could show that the second ATG (ATG2) is preferentially used in the wild-type constructs as well as in the K6 variant. This implies that the MC3R protein is shorter (37 amino acids) than initially predicted and that the T6K polymorphism is not located in the coding sequence.

In eucaryotic cells, the pre-initiation complex assembles at the cap structure at the $3^{\prime}$ end of the mRNA strand and moves in $5^{\prime}$ direction to the first AUG codon where translation is initiated [20], but AUG codons can also be bypassed by the ribosome. The context surrounding the AUG codon affects the translation initiation. A consensus sequence has been described as GCCRCCAUGG (R being a purine, the bold letters having the highest influence, and the start AUG indicated in italic) [21, 22]. In the human MC3R the sequence surrounding the first and the second AUG is GATTGAAUGA and ACAGCAAUGA, respectively. In concordance with our experiments the second AUG has a higher degree of sequence alignment with the optimal consensus sequence (indicated by underlined letters). In an analysis of over 2,000 human transcripts, it has been shown that more than $40 \%$ have additional upstream AUG codons [23].

Our data indicate that the hMC3R has an untranslated upstream AUG which is skipped probably by leaky scanning mechanisms. Furthermore, we showed that a common variant C17A (T6K) does not influence the translation initiation in vitro; however, we cannot rule out that C17A influences mRNA stability. This underlines the lack of association of this variant with obesity and related diseases in humans in most studies $[10,12,13]$. 
Tarnow et al.: hMC3R Translation Start

\section{Acknowledgements}

This work was funded by the Deutsche Forschungsgemeinschaft (BI 893/2-1) and graduate collegue 1208: Hormonal Regulation of Energy Metabolism, Body Weight and Growth, TP1 and the Bundesministerium für Bildung und Forschung NGFN ${ }^{\text {Plus }}$ (01GS825).

\section{Disclosure Statement}

The authors have nothing to disclose.

\section{References}

1 Huszar D, Lynch CA, Fairchild-Huntress V, Dunmore JH, Fang Q, Berkemeier LR, Gu W, Kesterson RA, Boston BA, Cone RD, Smith FJ, Campfield LA, Burn P, Lee F: Targeted disruption of the melanocortin-4 receptor results in obesity in mice. Cell 1997;88:131-141.

2 Butler AA, Kesteson RA, Khong K, Cullen MJ, Pelleymounter MA, Dekoning J, Baetscher M, Cone RD: A unique metabolic syndrome causes obesity in the melanocortin-3 receptor-deficient mouse. Endocrinology 2000;141:3518-3521.

3 Chen AS, Marsh DJ, Trumbauer ME, Frazier EG, Guan XM, Yu H, Rosenblum CI, Vongs A, Feng Y, Cao L, Metzger JM, Strack AM, Camacho RE, Mellin TN, Nunes CN, Min W, Fisher J, Gopal-Truter S, MacIntyre DE, Chen HY, Van der Ploeg LHT: Inactivation of the mouse melanocortin-3 receptor results in increased fat mass and reduced lean body mass. Nat Genet 2000;26:97-102.

- 4 Farooqi IS, Keogh JM, Yeo GSH, Lank EJ, Cheetham T, O’Rahilly S: Clinical spectrum of obesity and mutations in the melanocortin 4 receptor gene. N Engl J Med 2003;348:1085-1095.

- 5 Calton MA, Ersoy BA, Zhang S, Kane JP, Malloy MJ, Pullinger CR, Bromberg Y, Pennacchio LA, Dent R, McPherson R, Ahituv N, Vaisse C: Association of functionally significant melanocortin-4 but not melanocortin-3 receptor mutations with severe adult obesity in a large North American case control study. Hum Mol Genet 2009;18:1140-1147.

6 Lee YS, Poh LK-S, Loke KY: A novel melanocortin 3 receptor gene (MC3R) Mutation associated with severe obesity. J Clin Endocrinol Metab 2002;87:1423-1426.

7 Rached M, Buronfosse A, Begeot M, Penhoat A: Inactivation and intracellular retention of the human I183N mutated melanocortin 3 receptor associated with obesity. Biochim Biophys Acta 2004;1689:229-234.

- 8 Tao YX: Functional characterization of novel melanocortin-3 receptor mutations identified from obese subjects. Biochim BiophysActa 2007;1772:1167-1174.

- 9 Mencarelli M, Walker GE, Maestrini S, Alberti L, Verti B, Brunani A, Petroni ML, Tagliaferri M, Liuzzi A, Di Blasio AM: Sporadic mutations in melanocortin receptor 3 in morbid obese individuals. Eur J Hum Genet 2008;16:581-586.

$\checkmark 10$ Li W, Joo E, Furlong E, Galvin M, Abel K, Bell C, Price R: Melanocortin 3 receptor (MC3R) gene variants in extremely obese women. Int J Obes Relat Metab Disord 2000;24:206-210.

11 Schalin-Jantti C, Valli-Jaakola K, Oksanen L, Martelin E, Laitinen K, Krusius T, Mustajoki P, Heikinheimo M, Kontula K: Melanocortin-3-receptor gene variants in morbid obesity. Int J Obes Relat Metab Disord 2003; 27:70-74.

12 Hani EH, Dupont S, Durand E, Dina C, Gallina S, Gantz I, Froguel P: Naturally occurring mutations in the melanocortin receptor 3 gene are not associated with type 2 diabetes mellitus in French Caucasians. J Clin Endocrinol Metab 2001;86:2895-2898.

13 Wong J, Love DR, Kyle C, Daniels A, White M, Stewart AW, Schnell AH, Elston RC, Holdaway IM, Mountjoy KG: Melanocortin-3 receptor gene variants in a Maori kindred with obesity and early onset type 2 diabetes. Diabetes Res Clin Pract 2002;58:61-71.

14 Feng N, Young SF, Aguilera G, Puricelli E, Adler-Wailes DC, Sebring NG, Yanovski JA: Co-occurrence of two partially inactivating polymorphisms of MC3R is associated with pediatric-onset obesity. Diabetes 2005; 54:2663-2667.

15 Savastano DM, Tanofsky-Kraff M, Han JC, Ning C, Sorg RA, Roza CA, Wolkoff LE, Anandalingam K, JeffersonGeorge KS, Figueroa RE, Sanford EL, Brady S, Kozlosky M, Schoeller DA, Yanovski JA: Energy intake and energy expenditure among children with polymorphisms of the melanocortin-3 receptor. Am J Clin Nutr 2009;90:912-920.

16 Santoro N, Perrone L, Cirillo G, Raimondo P, Amato A, Brienza C, del Giudice EM: Effect of the melanocortin-3 receptor C17A and G241A variants on weight loss in childhood obesity. Am J Clin Nutr 2007;85: 950-953. 
17 Tao YX, Segaloff DL: Functional characterization of melanocortin-3 receptor variants identify a loss-offunction mutation involving an amino acid critical for G protein-coupled receptor activation. J Clin Endocrinol Metab 2004;89:3936-3942.

-18 Grosse J, Tarnow P, Römpler H, Schneider B, Sedlmeier R, Huffstadt U, Korthaus D, Nehls M, Wattler S, Schöneberg T, Biebermann H, Augustin M: N-ethyl-N-nitrosourea-based generation of mouse models for mutant G protein-coupled receptors. Physiol Genomics 2006;26:209-217.

-19 Engemaier E, Römpler H, Schöneberg T, Schulz A: Genomic and supragenomic structure of the nucleotidelike G-protein-coupled receptor GPR34. Genomics 2006;87:254-264.

20 Gebauer F, Hentze MW: Molecular mechanisms of translational control. Nat Rev Mol Cell Biol 2004;5:827835.

21 Kozak M: Possible role of flanking nucleotides in recognition of the AUG initiator codon by eukaryotic ribosomes. Nucleic Acids Res 1981;9:5233-5252.

22 Kozak M: Compilation and analysis of sequences upstream from the translational start site in eukaryotic mRNAs. Nucleic Acids Res 1984;12:857-872.

23 Peri S, Pandey A: A reassessment of the translation initiation codon in vertebrates. Trends Genet 2001;17: 685-687. 